\title{
Defining delirium in idiopathic Parkinson's disease: a systematic review
}

Rachael A Lawson ${ }^{1,2}$, Claire McDonald ${ }^{1,3}$, David J Burn ${ }^{4}$

1. Institute of Neuroscience, Newcastle University, UK

2. Newcastle University Institute for Ageing, Newcastle University, UK

3. Gateshead Health NHS Foundation Trust, UK

4. Faculty of Medical Science, Newcastle University, UK

Corresponding author: Rachael A Lawson

Clinical Ageing Research Unit

Institute of Neuroscience

Newcastle University Institute for Ageing

Newcastle University

Campus for Ageing and Vitality

Newcastle upon Tyne

NE4 5PL

01912081277

rachael.lawson@ncl.ac.uk

Word count: 3520

Figures: 1

Tables: 3

Running title: Defining delirium in idiopathic Parkinson's disease

Key words: Parkinson's disease, delirium, prevalence, systematic review. 


\begin{abstract}
Background:

Parkinson's disease patients may be at increased risk of delirium and developing adverse outcomes, such as cognitive decline and increased mortality. Delirium is an acute state of confusion that has overlapping symptoms with Parkinson's dementia, making it difficult to identify. This study aimed to determine the diagnostic criteria, prevalence, management strategies and outcomes of delirium in Parkinson's through a systematic review of the literature.
\end{abstract}

\title{
Methods:
}

Seven databases were used to identify all articles published before February 2017 comprising two key terms: "Parkinson's Disease" and "delirium". Data were extracted from studies meeting predefined inclusion criteria.

\section{Results:}

Twenty articles were identified. Delirium prevalence in Parkinson's ranged from 0.3-60\% depending on setting; a diagnosis of Parkinson's was associated with an increased risk of developing delirium. Delirium was identified/diagnosed using seven different criteria. Delirium may be associated with an increased length of hospital stay and worsening motor symptoms. We did not identify any studies examining the management of delirium in Parkinson's.

\section{Discussion:}

This review highlights the paucity of well-designed, appropriately powered studies investigating delirium in Parkinson's. The results suggest that delirium is a significant issue in people with Parkinson's and that having delirium may be a risk factor for adverse outcomes, particularly in inpatient settings. Further prospective research is needed to accurately determine the prevalence of delirium in Parkinson's, its management strategies and outcomes, and to evaluate diagnostic criteria to differentiate between the overlapping symptoms of Parkinson's and delirium. 


\section{Introduction}

Parkinson's disease (PD) is a neurodegenerative disorder defined by motor symptoms. However, it is now recognised that PD comprises a number of non-motor symptoms, which include neuropsychiatric features such as hallucination, delusions, anxiety and depression.[1] Cumulatively, $80 \%$ of patients with PD will develop dementia (PDD);[2] this is associated with chronic impaired but fluctuating attention, psychotic symptoms and delusions.[3] In addition, it is suspected that PD patients with and without dementia are at increased risk of delirium.[4]

Delirium is an acute neuropsychiatric syndrome that is common in older adults admitted to hospital.[5] Delirium is associated with an altered level of consciousness, confusion and impaired attention.[6] Delirium can be distressing for both the person with delirium and their families. Delirium in older adults has been associated with both short term and long term adverse consequences, such as increased risk of falls, cognitive decline, nursing home placement and higher rates of death. $[7,8]$ Occurrence of delirium is also associated with prolonged hospital stay[9] and higher costs per patient per admission.[5] In the only population-based study exploring the impact of delirium on cognitive outcomes, delirium was associated with an eight-fold increased risk of future dementia.[10] However, delirium may be preventable in a third of cases.[11, 12] Therefore, early detection and diagnosis could lead to improved outcomes for patients and reduced hospital costs.

There may be an overlapping clinical phenotype of PD and delirium. Commonly reported PD symptoms, such as attentional dysfunction, cognitive fluctuations, hallucinations, sleep disturbance and daytime somnolence are also common in delirium, $[4,6]$ making it difficult to distinguish PD symptoms from delirium. Therefore, previous studies may have either underestimated or overestimated the prevalence of delirium in people with PD.

Additionally, the management of delirium in PD is complex. Dopaminergic medication may predispose to delirium; neuroleptic medication, often used to treat delirium in older adults, may worsen motor symptoms resulting in increased rigidity and bradykinesia, which in turn can cause falls, impaired swallowing, aspiration and dehydration. 
Patients with PD may be at increased risk of developing delirium and adverse outcomes. Understanding the relationship between delirium and PD could help guide clinical practice. The aim of this systematic review was to: i) determine how delirium has been defined/diagnosed in PD patients in previous studies, ii) describe common presentations of delirium in people with PD, iii) establish the therapeutic strategies used to manage delirium in PD, and iv) determine the outcomes of delirium in people with PD.

\section{Methods}

\section{Search strategy}

We searched seven databases: Medline, Embase, PsychINFO, Cochran, Scopus, Web of Knowledge and the Cumulative Index to Nursing and Allied Health Literature (CINAHL). The search comprised two key terms: "Parkinson's Disease" and "delirium". For each key term, a list of synonyms were compiled (Table 1); where available, MeSH terms were used. The search included all articles published before February 2017. In addition, we examined references of articles from the search for articles not identified in the searches, but may be suitable for inclusion.

A central database was compiled using articles from the search; RAL removed all duplicates. Reviewers (RAL and CM) reviewed the titles and abstracts independently. The full text was reviewed where it was unclear whether the paper met inclusion criteria.

\section{Inclusion and exclusion criteria}

Articles were included if PD and delirium were referred to in the title or abstract, the paper was written in English and the full text was available. Articles were excluded if they included subjects with non-idiopathic parkinsonism, including multiple system atrophy, progressive supranuclear palsy, dementia with Lewy bodies (DLB), vascular PD, drug induced PD, subjects with a diagnosis of PD for less than one year, and could therefore meet the criteria for DLB, or where subjects had a history of brain injury or brain tumour. Review articles and case series were excluded. 


\section{Data extraction}

A data extraction form was generated which included study setting, study population, how participants were selected/identified, duration of study and follow up period. Key outcomes were also included on the data extraction form, including how delirium was diagnosed, the prevalence of delirium in PD, presenting symptoms of delirium, how delirium was managed and clinical outcomes associated with delirium, for example, duration of hospital admission, change in motor symptoms or cognitive function and mortality.

\section{Results}

The database searches identified 5672 potentially relevant articles ( Figure 1). After duplicates were removed ( $n=1278), 4394$ articles were identified. The title and abstract of the potentially relevant articles were screened and 4337 were excluded. We reviewed the full text of the remaining 56 articles. Thirty-nine articles were excluded because: the full text was not available in English ( $n=4)$, they were a conference abstract and no full text was available ( $n=19)$, the key outcomes identified by this systematic review were not available from the papers $(n=15)$, or the paper was a systematic review $(n=1)$. In addition to the 17 papers identified in the search which met inclusion criteria, three additional papers were identified by reviewers [13-15] by reviewing references of articles from the search and a previous review, [4] yielding a total of 20 papers which were eligible for data extraction (Table 2).

\section{Characteristics of studies and participants}

Of the 20 studies identified, ten were prospective, nine were retrospective and one was a case-control study (Table 2). The majority of studies were in an inpatient setting $(n=17)$ with two studies conducted in outpatients[16, 17] and one in residential and nursing homes.[18]

The samples ranged in size from 15-182,859 subjects with PD.[14, 19] The largest studies were retrospective review of medical databases.[14, 20,21] Older adults were the focus of the research in three studies, of which a proportion of subjects had PD.[18, 22, 23] Eight studies included postoperative subjects with PD (Table 2), five of which included PD patients who had undergone deep brain stimulation (DBS) surgery.[13, 19, 24-26] 
Participants with PDD were included in eight studies[14, 15, 18, 20, 22, 23, 27, 28] but were excluded from seven (Table 2).[13, 24-26, 29-31] The remaining studies did not give explicit details as to whether PDD participants were included in the study.[16, 17, 19, 21, 32]

\section{Defining and diagnosing delirium}

The criteria used to diagnose or identify delirium varied between studies (Table 2). Three studies used validated diagnostic criteria to diagnose delirium (Table 3). These comprised the Diagnostic and Statistical Manual of Mental Disorders (DSM-IV)[16, 28] and the International Classification of Diseases $10^{\text {th }}$ Revision (ICD-10).[14] Three studies used the Confusion Assessment Method (CAM), a validated assessment tool designed to allow nonspecialists to identify delirium.[22, 30, 31] In addition, Boorsma, et al. [18] used an adjusted version of the Nursing Home-CAM retrospectively by matching data from the Resident Assessment Instrument, a database used in Dutch nursing and residential care. However, the papers did not specify how these criteria were applied and what methods were used to assess specific criteria, such as attention.

The definition of delirium varied more widely between the remaining studies. Delirium was diagnosed by expert opinion by Golden, et al. [29] and Woodford and Walker.[15] Broader definitions of delirium were employed, which comprised "transient post-operative confusion"[19] and any event of hallucinations, delusions, or disorientation to circumstance.[24] Lange, et al. [25] defined delirium as "an altered mental state of reduced cooperation due to fear, psychomotor agitation and impaired or lost orientation." Oichi, et al. [21] used the prescription of antiparkinsonian medication as a marker for PD, and classified patients as having delirium if they were newly prescribed antipsychotic drugs postoperatively, which included quetiapine, risperidone, trazodone, mianserin and haloperidol. Six studies did not explicitly state how delirium was defined or diagnosed.[13, $17,20,26,27,32]$

\section{Prevalence of delirium}

The prevalence of delirium varied between studies and ranged from 0.3-60\% (Table 2). This likely reflects the variation in study design including setting, patient populations and definitions of delirium used across studies. Studies reporting primary and secondary reasons 
for hospital admissions reported the lowest prevalence rates $(0.3-5 \%) .[14,15]$ This method most likely underestimates the prevalence of delirium. Delirium is frequently secondary to an acute illness such as infections or hip fractures. The acute illness triggering delirium is more likely to be reported as the reason for admission rather than the concurrent delirium. This is demonstrated by the higher rates of delirium reported among medical inpatients. Studies that prospectively reviewed patients to identify delirium or retrospectively reviewed case notes for evidence of delirium reported prevalence of $22-48 \%$. Similarly, studies that assessed the prevalence of delirium in PD patients undergoing surgery reported prevalence rates of $11-60 \%$ in PD subjects (Table 2). $[13,19,22,24-26,29]$ The prevalence of delirium was lower in patients undergoing DBS (11-27\%) compared to other surgeries (22-60\%).

Delirium prevalence in outpatient settings was lower at 4\%.[16, 17] This likely reflects a population among which acute undercurrent illness was less common.

The prevalence was unclear in some studies due to the indistinct definitions of delirium applied.[20, 27] The prevalence of delirium in PD could not be ascertained by two studies,[22, 31] although Oh, et al. [22] reported that having PD was associated with a threefold increased risk of developing delirium (Table 2). Three other studies suggested having PD was associated with an increased risk of delirium.[18, 20, 21] Subjects with PD were five times as likely to be treated for delirium in inpatient settings,[20] and residents in a nursing home with PD were more than twice as likely to suffer from delirium.

Postoperatively, Oichi, et al. [21] found delirium was 8 times more likely in patients with PD undergoing spinal surgery than patients without PD undergoing the same surgery.

\section{Symptoms of delirium}

Eleven studies presented findings pertaining to symptoms of delirium in PD subjects.[16, 17, 19, 24-26, 28-32] Confusion and disorientation were the most commonly reported symptoms of delirium.[19, 25, 29, 31, 32]. Hallucinations and/or delusions were also commonly reported symptoms, $[16,17,24,29]$ although the two symptoms were not necessarily differentiated between. Golden, et al. [29] reported hallucinations in $36 \%$ of PD participants following DBS. Hallucinations were reported in all PD subjects with delirium by Nishikawa, et al. [17], but it was not clear whether hallucinations were directly related to 
delirium. Disturbances and fluctuations in consciousness, cognition and perception were symptoms associated with delirium.[28, 29] Golden, et al. [29] was the only study which reported day-night reversal although this was only in one subject. However, studies did not systematically assess or report these symptoms, thus it was not possible to draw conclusions as to their frequency.

Three studies hinted at delirium phenotype. Serrano-Duenas and Bleda [31] identified 76\% of PD subjects with delirium as "agitated type" delirium, while Lange, et al. [25] and Schupbach, et al. [26] reported that a small proportion of subjects ( $n=5$ and $n=3$, respectively) had postoperative hypomania. However, this should be viewed with caution, as hyperactive delirium was not explicitly stated. Of the remaining studies, a description of symptoms of delirium were either unclear or not stated.

\section{Outcomes of delirium}

Accurately defining the outcomes of delirium in patients with PD is challenging due to the limited information available from only nine studies (Table 2).[17, 24, 25, 27-32] Duration of delirium in PD patients ranged from 24 hours or less, to under 30 days.[17, 25, 29] Two studies showed that delirium was associated with extended hospital stay compared to PD patients without delirium. $[24,27]$

Longitudinal studies suggested that delirium could be associated with a worsening of motor symptoms in PD patients.[28, 31] Umemura, et al. [28] showed that patients with delirium were more than 15 times more likely to have motor deterioration $(O R=15.9)$, while SerranoDuenas and Bleda [31] showed that subjects with PD and delirium had significantly poorer motor scores compared to PD subjects without delirium after five years. Gerlach, et al. [32] reported that patient complications while hospitalised, which included delirium, were associated with a decline in motor symptoms, but the contribution of delirium could not be determined.

Whether delirium was associated with future PDD could not be ascertained. Serrano-Duenas and Bleda [31] found delirium was significantly associated with a decline in cognitive function over five years and increased mortality rates compared to age-matched PD patients without delirium and controls. However, these results should be viewed with 
caution due to the small sample size used (21 PD, 21 PD with delirium and 21 controls), and does not take into account additional hospital admissions or episodes of delirium. In addition, none of the studies assessed interventions for delirium or its associated outcomes.

\section{Discussion}

Key findings of this review include that the prevalence of delirium in PD varied widely between studies and settings, ranging from $0.3 \%$ to $60 \%$ of subjects, as did the criteria used to identify or diagnose delirium. In inpatient settings, delirium occurred in $11-60 \%$ patients and overall having PD was associated with an increased risk of developing delirium. Findings relating to symptoms and outcomes of delirium in PD patients were limited, but delirium may be associated with increased length of hospital stay and worsening motor symptoms.

The results showed that up to $60 \%$ of PD inpatients have delirium, and that having PD was a risk factor for developing delirium.[18, 20-22] However, given the wide range of reported prevalence of delirium in PD, some caution should be made when interpreting these results. The variation likely reflects the heterogeneous samples, settings and criteria used to define delirium in PD subjects. Firstly, the sample and study setting varied across studies and included both large retrospective studies and smaller prospective studies. As expected, delirium prevalence was lower in outpatient settings (4\%) compared to inpatient settings (22-48\%) and postoperatively (11-60\%). The proportion of PD patients whose primary reason for admission to hospital was delirium was low (0.3-5\%). This likely reflects current coding practices, where the underlying condition leading to delirium is documented as the primary reason for admission; however, this underestimates the proportion of patients whose admission is complicated by delirium. It also does not reflect cases were delirium developed while in hospital.[24].

A number of studies excluded patients with PDD; PDD is common and develops in up to $80 \%$ of patients.[2] As people with PDD may be most at risk of delirium,[18] a key group of people are likely to have been under investigated in previous research. None of the studies stratified for dementia; it is therefore difficult to determine the extent to which PDD or PD alone is a risk factor for delirium. Vida, et al. [23] showed that in non-demented older adults, participants with delirium had poorer instrumental activities of daily living (ADL) 
after 18 months compared to those without delirium, but there was no difference in functional outcomes in demented participants. This may reflect the pre-existing impact of dementia on ADL.

Nine different criteria were to used identify or diagnose delirium, and in six studies no methods were stated. Only seven studies used existing diagnostic criteria or a validated diagnostic tool to identify delirium in PD. Other studies used broader definitions of delirium, which included symptoms such as confusion or presence of hallucinations. Only a few studies referred to fluctuating cognition, but it was not clear whether this was a feature of cognitive impairment associated with PD or delirium. Use of objective measures, such as repeated cognitive tests, e.g. the Mini Mental State Examination, to determine change in cognition or improvement over time were not reported in the articles. Finally, the validity of the assessment tools, such as CAM, used by non-specialists to identify delirium have not been validated in PD. These issues have the potential to misidentify delirium as these symptoms are not uncommon in $\mathrm{PD},[3]$ and several articles were unclear whether these were acute changes or longstanding symptoms associated with PD.

Only limited inference could be made about the repercussions of delirium in PD patients. Increased length of hospital stay was associated with delirium in PD in two studies. This is consistent with studies in older adults, where occurrence of delirium has also been associated with prolonged hospital admission[9] and higher costs per patient per admission.[5] However, duration of hospital admission can be influenced by other factors, such as comorbidities and frailty, which are also risk factors for delirium.

An increasing number of studies have reported that delirium is a predictor of dementia and is associated with worsening of existing cognitive decline.[10, 33-35] Only one study has examined the association between delirium and cognitive decline in PD. Serrano-Duenas and Bleda [31] found that PD patients with delirium were at greater risk of cognitive decline five years later. This is in keeping with studies in the general population. A population-based study in older adults found that delirium was associated with an eight-fold increased risk of future dementia.[10] A systematic review in older adults reported that delirium was associated with increased mortality, functional outcomes and institutionalisation.[9] Further research replicating these findings in PD patients is needed. 
There are some limitations to be acknowledged. We used a broad search strategy but excluded articles not available in English; however, we believe this review is comprehensive and includes the key findings in this area. The quality and methodological rigour differed between studies. Although some studies used the Queen's Square Brain Bank criteria to identify idiopathic PD, this was not always possible due to the methodology of some studies, for example in retrospective studies. One study used prescribed anti-parkinsonian medication as a surrogate marker for PD,[21] however, this could inadvertently include patients with other parkinsonian conditions and patients who used dopaminergic therapy, for other indications such as restless legs syndrome. Retrospective studies of hospital databases and review of medical notes has the advantage of accessing large amounts of existing data. However, it has been shown that medical records are unreliable sources of delirium as delirium is under-diagnosed, inconsistently documented and underreported.[36] Furthermore, as demonstrated in one paper,[20] delirium and encephalopathy can be used interchangeably; therefore, delirium could be misreported in some subjects. Due to the limited number of studies whose focus was delirium in PD, we used broad criteria to identify and consolidate all currently published literature in this area. However, our findings highlights the need of future studies to use validated criteria to verify the diagnosis of idiopathic PD, such as the Queen's Square Brain Bank criteria, and delirium, such as DSM-5 criteria or CAM.

Most noticeably, we did not find any studies examining interventions to prevent or treat delirium in PD. The dopaminergic and cholinergic deficits in PD theoretically make patients more vulnerable to delirium and it remains to be established if conservative measures shown to prevent delirium in general older adults are as effective in PD.[11, 12] Furthermore, there are specific challenges to managing delirium in PD. Many of the medications prescribed to PD patients are associated with increased risk of delirium, and guidelines suggest a medication review should form part of the management of delirium in PD.[37]

A first approach should be to identify and treat the underlying precipitant (e.g. infection, dehydration etc.). Where conservative measures and treating the underlying precipitant fails, clinicians may be required to alter or suspend PD medication. The consensus is that drugs with the greatest anticholinergic effect should be removed first. [4] Thus, PD 
medication could be stopped or suspended in the following order: anticholinergics, amantadine, monoamine oxidase inhibitors, dopamine agonists, levodopa. A review of the current evidence, however, shows these guidelines are not based on hard evidence but clinical experience.[4] It must be noted that the sudden withdrawal of dopaminergic medication could increase the risk of patients developing delirium.

Antipsychotic medications are used in patients without PD to control delirium; however, a meta-analysis of randomised controlled trials (RCTs) failed to show any impact of antipsychotics on duration or severity of delirium, or on length of stay or mortality.[38] Most antipsychotics can worsen motor symptoms in PD and are, therefore, relatively contraindicated. Where neuroleptic mediation is necessary, quetiapine is considered the safest choice; however, there are no studies examining its use in PD.[4] Other agents have been considered for delirium, but not specifically in PD. RCTs of cholinesterase inhibitors in older adults have failed to show superiority compared to placebo, and some have suggested increased adverse events in the treatment arm of these studies.[39] Melatonin has been associated with a decreased incidence of delirium in RCTs performed in older patients on medical wards, but not in the surgical setting. [40]

In summary, this review highlights that there is a paucity of well-powered studies investigating delirium in PD and its outcomes. Nonetheless, the results suggest that delirium is a significant issue in people with PD; it may occur more frequently in PD than in older adults, particularly in inpatient settings and postoperatively. Given its potential association with worsening PD motor symptoms, cognitive decline and increased mortality rates, it is important that delirium is regularly screened for and its diagnosis recorded appropriately. More research is warranted in this area. We propose that future studies should consider using prospective methods to investigate the prevalence of delirium in PD, its outcomes and management strategies. Studies that accurately characterise delirium in PD and evaluate diagnostic criteria may also be useful to clinicians and researchers to differentiate between the overlapping symptoms of PD and delirium. A better understanding of delirium and its presentation in PD will be important for treatment selection in future clinical trials to treat or prevent delirium. 


\section{Declaration of interest}

None.

\section{Funding}

This research did not receive any specific grant from funding agencies in the public, commercial, or not-for-profit sectors. Dr Lawson is funded by Parkinson's UK and has previously been supported by the Lockhart Parkinson's Disease Research Fund. Dr McDonald is funded by an NIHR clinical lectureship. Professor Burn has previously held grants by Parkinson's UK.

\section{Author roles}

Dr Lawson was involved with study conception and design, organisation, execution, data search, reviewed manuscripts for data extraction, analysis and interpretation of data and preparation of the manuscript.

Dr McDonald was involved with study design and organisation, execution, data search, reviewed manuscripts for data extraction, analysis and interpretation and preparation of the manuscript.

Professor Burn was involved with study conception and design, analysis and interpretation of data and preparation of the manuscript.

\section{References}

[1] R. Balestrino, P. Martinez-Martin, Neuropsychiatric symptoms, behavioural disorders, and quality of life in Parkinson's disease, J Neurol Sci 373 (2017) 173-178.

[2] M.A. Hely, W.G. Reid, M.A. Adena, G.M. Halliday, J.G. Morris, The Sydney multicenter study of Parkinson's disease: the inevitability of dementia at 20 years, Mov Disord 23(6) (2008) 837-44.

[3] I. Leroi, H. Pantula, K. McDonald, V. Harbishettar, Neuropsychiatric symptoms in Parkinson's disease with mild cognitive impairment and dementia, Parkinsons Dis 2012 (2012) 308097. 
[4] E.R. Vardy, A. Teodorczuk, A.J. Yarnall, Review of delirium in patients with Parkinson's disease, J Neurol 262(11) (2015) 2401-10.

[5] S.K. Inouye, R.G. Westendorp, J.S. Saczynski, Delirium in elderly people, Lancet 383(9920) (2014) 911-22.

[6] American Psychiatric Association, The Diagnostic and Statistical Manual of Mental Disorders: DSM 5, American Psychiatric Association, Washington, DC, 2013.

[7] K. Rockwood, S. Cosway, D. Carver, P. Jarrett, K. Stadnyk, J. Fisk, The risk of dementia and death after delirium, Age Ageing 28(6) (1999) 551-556.

[8] B.E. Lakatos, V. Capasso, M.T. Mitchell, S.M. Kilroy, M. Lussier-Cushing, L. Sumner, J. Repper-Delisi, E.P. Kelleher, L.A. Delisle, C. Cruz, T.A. Stern, Falls in the General Hospital: Association With Delirium, Advanced Age, and Specific Surgical Procedures, Psychosomatics 50(3) (2009) 218-226.

[9] N. Siddiqi, A.O. House, J.D. Holmes, Occurrence and outcome of delirium in medical inpatients: a systematic literature review, Age Ageing 35(4) (2006) 350-364.

[10] D.H. Davis, G. Muniz Terrera, H. Keage, T. Rahkonen, M. Oinas, F.E. Matthews, C. Cunningham, T. Polvikoski, R. Sulkava, A.M. MacLullich, C. Brayne, Delirium is a strong risk factor for dementia in the oldest-old: a population-based cohort study, Brain 135(Pt 9) (2012) 2809-16.

[11] S.K. Inouye, S.T. Bogardus, Jr., P.A. Charpentier, L. Leo-Summers, D. Acampora, T.R. Holford, L.M. Cooney, Jr., A multicomponent intervention to prevent delirium in hospitalized older patients, N Engl J Med 340(9) (1999) 669-76.

[12] Y.C. Youn, H.W. Shin, B.S. Choi, S. Kim, J.Y. Lee, Y.C. Ha, Rivastigmine patch reduces the incidence of postoperative delirium in older patients with cognitive impairment, Int J Geriatr Psychiatry 10.1002/gps.4569 (2016).

[13] P. Krack, A. Batir, N. Van Blercom, S. Chabardes, V. Fraix, C. Ardouin, A. Koudsie, P.D. Limousin, A. Benazzouz, J.F. LeBas, A.-L. Benabid, P. Pollak, Five-Year Follow-up of Bilateral 
Stimulation of the Subthalamic Nucleus in Advanced Parkinson's Disease, N Engl J Med 349(20) (2003) 1925-1934.

[14] V. Low, Y. Ben-Shlomo, E. Coward, S. Fletcher, R. Walker, C.E. Clarke, Measuring the burden and mortality of hospitalisation in Parkinson's disease: A cross-sectional analysis of the English Hospital Episodes Statistics database 2009-2013, Parkinsonism Relat Disord 21(5) (2015) 449-454.

[15] H. Woodford, R. Walker, Emergency hospital admissions in idiopathic Parkinson's disease, Mov Disord 20(9) (2005) 1104-8.

[16] S. Holroyd, L. Currie, G.F. Wooten, Prospective study of hallucinations and delusions in Parkinson's disease, J Neurol Neurosurg Psychiatry 70(6) (2001) 734-738.

[17] N. Nishikawa, M. Nagai, T. Moritoyo, H. Yabe, M. Nomoto, Plasma amantadine concentrations in patients with Parkinson's disease, Parkinsonism Relat Disord 15(5) (2009) 351-353.

[18] M. Boorsma, K.J. Joling, D.H. Frijters, M.E. Ribbe, G. Nijpels, H.P. van Hout, The prevalence, incidence and risk factors for delirium in Dutch nursing homes and residential care homes, Int J Geriatr Psychiatry 27(7) (2012) 709-715.

[19] E. Kahn, P.F. D'Haese, B. Dawant, L. Allen, C. Kao, P.D. Charles, P. Konrad, Deep brain stimulation in early stage Parkinson's disease: Operative experience from a prospective randomised clinical trial, J Neurol Neurosurg Psychiatry 83(2) (2012) 164-170.

[20] M. Lubomski, R.L. Rushworth, S. Tisch, Hospitalisation and comorbidities in Parkinson's disease: a large Australian retrospective study, J Neurol Neurosurg Psychiatry 86(3) (2015) 324-30.

[21] T. Oichi, H. Chikuda, J. Ohya, R. Ohtomo, K. Morita, H. Matsui, K. Fushimi, S. Tanaka, H. Yasunaga, Mortality and morbidity after spinal surgery in patients with Parkinson's disease: a retrospective matched-pair cohort study, Spine J 17(4) (2017) 531-537. 
[22] E.S. Oh, F.E. Sieber, J.M. Leoutsakos, S.K. Inouye, H.B. Lee, Sex Differences in Hip Fracture Surgery: Preoperative Risk Factors for Delirium and Postoperative Outcomes, J Am Geriatr Soc 64(8) (2016) 1616-1621.

[23] S. Vida, G.G. Du Fort, R. Kakuma, L. Arsenault, R.W. Platt, C.M. Wolfson, An 18-month prospective cohort study of functional outcome of delirium in elderly patients: Activities of daily living, Int Psychogeriatr 18(4) (2006) 681-700.

[24] J.D. Carlson, J.J. Neumiller, L.D. Swain, J. Mark, P. McLeod, J. Hirschauer, Postoperative delirium in Parkinson's disease patients following deep brain stimulation surgery, J Clin Neurosci 21(7) (2014) 1192-5.

[25] M. Lange, N. Zech, M. Seemann, A. Janzen, D. Halbing, F. Zeman, C. Doenitz, E. Rothenfusser, E. Hansen, A. Brawanski, J. Schlaier, Anesthesiologic regimen and intraoperative delirium in deep brain stimulation surgery for Parkinson's disease, J Neurol Sci 355(1-2) (2015) 168-173.

[26] W.M. Schupbach, N. Chastan, M.L. Welter, J.L. Houeto, V. Mesnage, A.M. Bonnet, V. Czernecki, D. Maltete, A. Hartmann, L. Mallet, B. Pidoux, D. Dormont, S. Navarro, P. Cornu, A. Mallet, Y. Agid, Stimulation of the subthalamic nucleus in Parkinson's disease: a 5 year follow up, J Neurol Neurosurg Psychiatry 76(12) (2005) 1640-4.

[27] C. Klein, T. Prokhorov, A. Miniovitz, E. Dobronevsky, J.M. Rabey, Admission of Parkinsonian patients to a neurological ward in a community hospital, J Neural Transm (Vienna) 116(11) (2009) 1509-1512.

[28] A. Umemura, T. Oeda, S. Tomita, R. Hayashi, M. Kohsaka, K. Park, H. Sugiyama, H. Sawada, Delirium and high fever are associated with subacute motor deterioration in parkinson disease: A nested case-control study, PLoS ONE 9 (6) (no pagination)(e94944) (2014).

[29] W. Golden, R.C. Lavender, W.S. Metzer, Acute postoperative confusion and hallucinations in Parkinson disease, Ann Intern Med 111(3) (1989) 218-222. 
[30] M.S. Kim, J.H. Yoon, H.J. Kim, S.W. Yong, J.M. Hong, Olfactory dysfunction is related to postoperative delirium in Parkinson's disease, J Neural Transm (Vienna) 123(6) (2016) 589594.

[31] M. Serrano-Duenas, M.J. Bleda, Delirium in Parkinson's disease patients. A five-year follow-up study, Parkinsonism Relat Disord 11(6) (2005) 387-392.

[32] O.H.H. Gerlach, M.P.G. Broen, W.E.J. Weber, Motor outcomes during hospitalization in Parkinson's disease patients: A prospective study, Parkinsonism Relat Disord 19(8) (2013) 737-741.

[33] T.G. Fong, R.N. Jones, P. Shi, E.R. Marcantonio, L. Yap, J.L. Rudolph, F.M. Yang, D.K. Kiely, S.K. Inouye, Delirium accelerates cognitive decline in Alzheimer disease, Neurology 72(18) (2009) 1570-5.

[34] A.M. MacLullich, A. Beaglehole, R.J. Hall, D.J. Meagher, Delirium and long-term cognitive impairment, Int Rev Psychiatry 21(1) (2009) 30-42.

[35] P.P. Pandharipande, T.D. Girard, J.C. Jackson, A. Morandi, J.L. Thompson, B.T. Pun, N.E. Brummel, C.G. Hughes, E.E. Vasilevskis, A.K. Shintani, K.G. Moons, S.K. Geevarghese, A. Canonico, R.O. Hopkins, G.R. Bernard, R.S. Dittus, E.W. Ely, Long-term cognitive impairment after critical illness, N Engl J Med 369(14) (2013) 1306-16.

[36] E. Kuhn, X. Du, K. McGrath, S. Coveney, N. O'Regan, S. Richardson, A. Teodorczuk, L. Allan, D. Wilson, S.K. Inouye, A.M.J. MacLullich, D. Meagher, C. Brayne, S. Timmons, D. Davis, Validation of a Consensus Method for Identifying Delirium from Hospital Records, PLoS ONE 9(11) (2014) e111823.

[37] National Institute for Health and Clinical Excellence, Delirium: prevention, diagnosis and management, 2010, p. Clinical Guideline 103.

[38] K.J. Neufeld, J. Yue, T.N. Robinson, S.K. Inouye, D.M. Needham, Antipsychotic Medication for Prevention and Treatment of Delirium in Hospitalized Adults: A Systematic Review and Meta-Analysis, J Am Geriatr Soc 64(4) (2016) 705-14. 
[39] J.I. Friedman, L. Soleimani, D.P. McGonigle, C. Egol, J.H. Silverstein, Pharmacological treatments of non-substance-withdrawal delirium: a systematic review of prospective trials, American Journal of Psychiatry 171(2) (2014) 151-159.

[40] S. Chen, L. Shi, F. Liang, L. Xu, D. Desislava, Q. Wu, J. Zhang, Exogenous melatonin for delirium prevention: a meta-analysis of randomized controlled trials, Molecular neurobiology 53(6) (2016) 4046-4053.

[41] American Psychiatric Association, Diagnostic and statistical manual of mental disorders: DSM-IV, American Psychiatric Association 1994.

[42] World Health Organization, ICD-10, the ICD-10 classification of mental and behavioural disorders: Diagnostic criteria for research, Geneva: World Health Organization (1993).

[43] S.K. Inouye, C.H. van Dyck, C.A. Alessi, S. Balkin, A.P. Siegal, R.I. Horwitz, Clarifying confusion: The confusion assessment methoda new method for detection of delirium, Ann Intern Med 113(12) (1990) 941-948.

Figure 1: PRISMA diagram presenting the search yield for the systematic review 